\title{
Comprensión del cambio psicoterapéutico en adolescentes: voces de pacientes y terapeutas
}

\author{
Understanding the psychotherapeutic change in adolescents: \\ Voices of patients and therapists
}

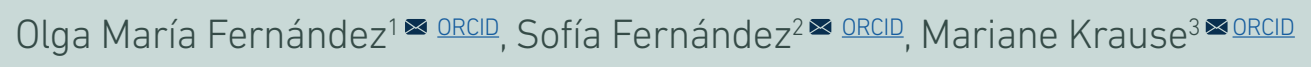

${ }^{1}$ Universidad de Chile

${ }^{2}$ Pontificia Universidad Católica de Chile

Fecha correspondencia:

Recibido: diciembre 2 de 2019.

Aceptado: septiembre 3 de 2020.

Forma de citar:

Fernández, 0., Fernández, S., \&

Krause, M. (2020). Comprensión

del cambio psicoterapéutico en

adolescentes: voces de pacientes

y terapeutas. Rev. CES Psico, 13(3),

107-123.

\section{Open access}

(c) Copyright

Licencia creative commons

Etica de publicaciones

Revisión por pares

Gestión por Open Journal System

DOI: http://dx.doi.org/10.21615/

cesp.13.3.7

ISSN: 2011-3080

Comparte

\section{Resumen}

Este estudio tuvo como objetivo comprender el proceso de cambio psicoterapéutico desde las perspectivas subjetivas de adolescentes y terapeutas que participaron en una terapia. Método: se utilizó un enfoque cualitativo; la recolección de información se realizó a través de entrevistas semiestructuradas audiograbadas, luego de finalizar la terapia. Participaron 17 díadas terapéuticas (adolescentes: edad promedio 15,8 años; 64,7\% género femenino; terapeutas: edad promedio 30,3 años; 55,5 \% género femenino), que realizaron sus terapias en contextos naturales y con diferentes enfoques teóricos. Codificadores entrenados analizaron los datos a través de los procedimientos propuestos por la Teoría Fundamentada, codificación abierta y axial, y procesos de reorganizaciones y triangulaciones permanentes. Resultados: se identificaron dos fenómenos comprensivos relacionados con los procesos de cambio psicoterapéutico: (a) El encuentro con "otro" como plataforma segura y sustrato relacional generador del cambio y, (b) El cambio como empoderamiento y desarrollo. Discusión: se examina el rol central que ocupan la relación terapéutica y el logro de las tareas del desarrollo (identidad, autonomía y autodeterminación) en el cambio psicoterapéutico. Además, se reflexiona acerca de las habilidades y acciones de los terapeutas.

Palabras claves: Psicoterapia con Adolescentes, Procesos de Cambio Psicoterapéutico, Alianza Terapéutica, Metodología Cualitativa.

\section{Abstract}

This study aimed to understand the process of psychotherapeutic change from the subjective perspectives of adolescents and therapists who participated in therapy. Method: A qualitative approach was used; information was collected through a semi-structured audio-recorded interview, which occurred at the end of therapy. The participants were 17 therapeutic dyads (adolescents: average age 15.8 years; $64.7 \%$ female gender; therapists: average age 30.3 years; $55.5 \%$ female gender), who performed their therapies in natural contexts and with different theoretical approaches. Trained 
Pág 108

\section{Sobre los autores:}

1. Doctora en Psicoterapia.

Psicóloga Clínica. Profesora

Asistente Departamento

Psiquiatría y Salud Mental

Facultad de Medicina

Universidad de Chile.

Investigadora Adjunta

Instituto Milenio para

la Investigación de la

Depresión y la Personalidad

(MIDAP).

2. Magíster en Psicología

Clínica. Psicóloga Clínica.

Estudiante de Doctorado

en Psicoterapia Pontificia

Universidad Católica de

Chile. Estudiante en Instituto

Milenio para la Investigación

de la Depresión y la

Personalidad (MIDAP).

3. Doctora en Psicología.

Psicóloga. Profesora

Titular, Escuela de

Psicología Pontificia

Universidad Católica

de Chile. Investigadora

Asociada Instituto Milenio

para la Investigación de la

Depresión y la Personalidad (MIDAP). coders analyzed the data through the procedures proposed by the Grounded Theory, with open and axial coding, performing processes of permanent reorganizations and triangulations. Results: The results show two major phenomena related to the processes of change: (a) The encounter with "the other" as a safe platform and relational substrate to generate change and (b) Change as empowerment and development. Discussion: The central role of the therapeutic relationship and the achievement of developmental tasks (identity, autonomy and self-determination) in psychotherapeutic change is discussed. In addition, it also reflects on the skills and actions of the therapists.

Keywords: Psychotherapy with Adolescents, Adolescent Therapy, Change Processes, Therapeutic Alliance, Qualitative Methods.

\section{Introducción}

La salud mental de la población adolescente constituye un desafío de alta relevancia en el ámbito de la salud pública mundial (Midgley et al., 2018). Se estima que entre $10 \%$ y $20 \%$ de los adolescentes en el mundo experimentan trastornos mentales, pero no en todos los casos se diagnostican ni se tratan adecuadamente (Kessler, et al., 2007); y en poblaciones vulnerables estas cifras pueden ser más altas. Se calcula que en América Latina y el Caribe por lo menos 53 millones de niños, niñas, adolescentes y jóvenes requieren atención en salud mental (Benjet, 2009). A su vez, la mayoría de los problemas de salud mental de la infancia y la adolescencia conllevan problemas en la vida adulta (Benjet, 2009; Kessler et al., 2005; Russel, 2008), con alto impacto en la calidad de vida de las personas y consecuencias que se traducen en costos económicos y productivos para la sociedad (Benjet, 2009; Russell, 2008).

La psicoterapia se ha constituido en una estrategia para responder a las necesidades de salud mental y contribuir al bienestar socioemocional de los jóvenes y de la población en general (Garcia, 2019; Weisz \& Kazdin, 2010). Cabe señalar que, la psicoterapia con adolescentes presenta características y complejidades específicas que la diferencian de la psicoterapia con adultos, por lo que no es recomendable la extrapolación del acervo teórico, las técnicas y los resultados de investigaciones en psicoterapia de un grupo etario a otro (DiGiuseppe, Linscott, \& Jilton, 1996; Russell, 2008). Existe evidencia de que los/as adolescentes presentan dificultad en el establecimiento de la relación de ayuda, se muestran reacios a la atención, manifiestan oposición activa o pasiva a consultar, tienen inconsistencias en su motivación, o simplemente desertan en las primeras sesiones de sus tratamientos (Fernández, Herrera, \& Escobar, 2016; Kazdin, 2004; Manríquez, Molina, \& Zubarew, 2003). También, se ha observado que, por lo general, los adolescentes asisten a tratamiento por motivación externa (padres, familia o escuela) y, con frecuencia, no perciben la disfuncionalidad de su conducta (Kazdin, 2004), por lo que llegan a la terapia sin motivación propia y sin un motivo de consulta personal. Además, en la terapia con adolescentes es común la participación directa o indirecta de los padres u otros miembros del grupo familiar, o de instancias educativas o judiciales; lo que disminuye la privacidad y fomenta la desconfianza de los adolescentes, así como su reticencia a recibir ayuda (Everall \& Paulson, 2002; Kazdin, 2004).

Las características de desarrollo propias de la edad adolescente parecen mediar parte de las singularidades descritas. La búsqueda de la identidad, junto con la necesidad de autonomía y autodeterminación, así como el proceso de separación-individuación en relación con los padres y figuras de autoridad, se relacionan con la desconfianza y rechazo a recibir ayuda de otros adultos (Levisky, 1999; Russell, Shirk, 
Pág 109

Existe evidencia consistente sobre la efectividad de la psicoterapia con adolescentes, tanto en la disminución de la sintomatología de diferentes trastornos, el aumento del nivel de adaptación y bienestar como en la recuperación del curso normal del desarrollo (Kazdin, 2004; Goodyer et al., 2011; Weisz \& Kazdin, 2010; Weisz, Sandler, Durlak, \& Anton, 2005).
\& Jungbluth, 2008). Así mismo, el surgimiento del pensamiento hipotético deductivo propio de la edad (Marchesi, Palacios, \& Coll, 2017), los encamina a relativizar la opinión de otros y a desidealizar o desvalorizar su aporte y apoyo. Además, se ha señalado que la dimensión narcisista, acentuada en la adolescencia, potencia que el/la adolescente se considere único/a, y esté convencido/a de que nadie puede comprenderlo y contenerlo: lo que puede interferir en el vínculo inicial con el tratamiento y provocar resistencia e interrupción de la terapia (Levisky, 1999). En este sentido, diversos autores (Everall \& Paulson, 2002; Páramo, 2011) plantean que la adolescencia requiere modelos de abordaje terapéutico diferentes a los de la niñez y adultez.

\section{Investigaciones en psicoterapia con adolescentes}

Existe evidencia consistente sobre la efectividad de la psicoterapia con adolescentes, tanto en la disminución de la sintomatología de diferentes trastornos, el aumento del nivel de adaptación y bienestar como en la recuperación del curso normal del desarrollo (Kazdin, 2004; Goodyer et al., 2011; Weisz \& Kazdin, 2010; Weisz, Sandler, Durlak, \& Anton, 2005). Sin embargo, muchas de estas investigaciones se han realizado en culturas anglosajonas y en contextos artificiales (experimentales), a través de estudios clínicos randomizados, con pacientes y procedimientos muy depurados (cuadros clínicos sin comorbilidades e intervenciones manualizadas) (Goodyer, et al., 2011; Weisz \& Kazdin, 2010); lo que dificulta la adaptación y aplicación de sus hallazgos a la práctica clínica en contextos naturales, y en otros entornos, por ejemplo, en Latinoamérica, donde la realidad sociocultural y sanitaria es diferente a la de los países anglosajones. Otra limitación de los estudios de efectividad es que se han centrado preferentemente en la evaluación de resultados finales, más que de los procesos generadores de los cambios; y la metodología de investigación empleada en la comprobación de la efectividad no muestra con claridad qué es aquello que efectivamente provoca el cambio. A su vez, la información de los resultados es reportada casi siempre por terceros (terapeutas, padres, profesores u otro adulto responsable), lo que invisibiliza las percepciones de los niños y adolescentes.

Un desafío aún pendiente es conocer los procesos o mecanismos que generan los cambios en psicoterapia con adolescentes (Kazdin, 2007, 2009). Los estudios que buscan responder cómo se produce el cambio, se han orientado especialmente al análisis de la Alianza Terapéutica o la relación terapéutica y, en menor proporción al lenguaje y las actividades realizadas durante la sesión terapéutica.

La relación terapéutica es un factor, directo o indirecto, que actúa sobre los resultados (Hoffat, Borge, Sexton, Clark, \& Wampold, 2012) y ha sido estudiada con prioridad, a partir del concepto Alianza Terapéutica (AT) (Bordin, 1976; Corbella, \& Botella, 2003; Horvath, 2001). Estudios con niños, adolescentes y adultos muestran alta correlación de la AT con los resultados de la psicoterapia (Martin, Garske \& Davies, 2000; Russell et al., 2008; Shirk \& Karver, 2003). El nivel de AT evaluada en las primeras sesiones, desde la perspectiva de los adolescentes, es el que presenta mayor relación con los resultados de la psicoterapia (Diamond, Siqueland, \& Diamond, 2003; Fernández, Krause, \& Pérez, 2016), y esta relación es moderada por la involucración del adolescente en su tratamiento (Karver, et al., 2008).

La AT también ha sido estudiada a partir de los conceptos de ruptura y reparación (Safran \& Muran, 2006). De manera particular, se ha encontrado que los adolescentes tienden a presentar eventos de ruptura en la psicoterapia, especialmente en los momentos iniciales y finales (Schenk et al., 2019); estos eventos se asocian a momentos 
Pág 110

Los adolescentes consideran la relación terapéutica como una experiencia central de la psicoterapia (Fernández et al., 2016; Everal \& Paulson, 2002; Páramo, 2011); tienden a iniciarla con escepticismo y reticencia, y luego la perciben muy diferente a otras relaciones con adultos: más igualitaria, confiable, respetuosa y comprensiva, con un alto componente afectivo positivo, homologada a una relación con un "amigo especial" (Everall \& Paulson, 2002; Fernández et al., 2016; Páramo, 2011). en los que el adolescente siente distanciamiento atencional y emocional de parte de los terapeutas (Morán, Díaz, Martínez, Varas, \& Sepúlveda, 2019), y su manejo es clave para el proceso terapéutico, en términos de adherencia y de resultados (Daly, Llewelyn, McDougall, \& Chanen, 2010).

Los adolescentes consideran la relación terapéutica como una experiencia central de la psicoterapia (Fernández et al., 2016; Everal \& Paulson, 2002; Páramo, 2011); tienden a iniciarla con escepticismo y reticencia, y luego la perciben muy diferente a otras relaciones con adultos: más igualitaria, confiable, respetuosa y comprensiva, con un alto componente afectivo positivo, homologada a una relación con un "amigo especial" (Everall \& Paulson, 2002; Fernández et al., 2016; Páramo, 2011).

Los estudios acerca de lenguaje o acciones comunicacionales en psicoterapia con adolescentes han mostrado que un lenguaje colaborativo (centrado en el "nosotros") es predictor de una AT positiva y de mejores resultados; mientras la presión para que el adolescente hable se relaciona negativamente con la AT y con los logros en la terapia (Creed \& Kendall, 2005). Por su parte, Krause, Fernández y Bräutigam (2015) señalan que la fase inicial de las terapias exitosas se caracteriza por interacciones verbales con mayor proporción de aseveraciones, intenciones de sintonizar con el otro y referencias a sí mismo y a la relación terapéutica, en comparación con las terapias sin cambios significativos. Este diálogo favorecedor del cambio es promovido por preguntas del terapeuta, que el/la adolescente percibe diferentes a las que se presentan en sus interacciones cotidianas (Everall \& Paulson, 2002). Otro estudio realizado por Fernández, Pérez y Krause (2019) indicó que, cuando los adolescentes perciben un vínculo fuerte se incrementan las probabilidades de que la conversación se centre en la construcción de nuevos significados; mientras que si perciben el énfasis en la dimensión "metas" se presenta mayor probabilidad de que el diálogo se dirija a buscar la sintonía entre terapeuta y paciente.

Existe evidencia de que la problemática adolescente se puede trabajar con éxito desde distintas orientaciones teóricas y técnicas (Páramo, 2011). Un metaanálisis realizado por Shirk y Karver (2003) confirma estos hallazgos al demostrar que la orientación teórica de la terapia, su modalidad (individual, familiar o solo a padres) y su nivel de estructuración (manualizada versus no manualizada) no son moderadores significativos en la asociación de la AT con los resultados; y del mismo modo se comportan la edad del paciente y el contexto de la terapia (servicio de atención en salud y contexto de investigación). En relación con la duración de las terapias, Páramo (2011) plantea que presentan mayor viabilidad y eficacia los procesos terapéuticos de corta duración, orientados a objetivos concretos, alcanzables y consensuados desde un inicio entre el adolescente y el terapeuta. $Y$ algunos reportes mencionan que el espacio físico cómodo y relajado, que los adolescentes sienten como propio, es valorado en sus experiencias terapéuticas (Everall, \& Paulson, 2002; Fernández et al., 2016; Páramo, 2011).

Las actitudes y habilidades de los terapeutas, que los jóvenes valoran de manera positiva son la aceptación e interés genuino del terapeuta por el paciente, su flexibilidad y creatividad, su capacidad directiva, de negociación y de ofrecer apoyo (Everall \& Paulson, 2002; Fernández et al., 2016; Páramo, 2011).

También se ha planteado que en las intervenciones con adolescentes se debe considerar la colaboración comprometida de los padres, para que el proceso de cambio ocurra (Binder, Holgersen, \& Nielsen, 2008; Fernández, 2019; Páramo, 2011). 
Pág 111

Este estudio utilizó un diseño cualitativo exploratorio descriptivo, de corte transversal, con entrevistas de seguimiento post terapia, para explorar en profundidad las experiencias subjetivas de adolescentes y terapeutas acerca de la generación del cambio en procesos terapéuticos.
Dadas las características particulares de la psicoterapia con adolescentes y considerando que la perspectiva de los adolescentes sobre su proceso psicoterapéutico ha sido poco investigada, este estudio busca responder al desafío de comprender el cambio terapéutico en adolescentes desde la experiencia subjetiva de los actores del proceso, en terapias realizadas en sus contextos naturales, pacientes con distintos diagnósticos y terapeutas con distintos enfoques teóricos.

El objetivo del estudio es, entonces, generar un modelo comprensivo del proceso de cambio en psicoterapia con adolescentes, que enfatice las relaciones y los mecanismos atribuidos subjetivamente por adolescentes y terapeutas como generadores de los cambios.

\section{Método}

Este estudio utilizó un diseño cualitativo exploratorio descriptivo, de corte transversal, con entrevistas de seguimiento post terapia, para explorar en profundidad las experiencias subjetivas de adolescentes y terapeutas acerca de la generación del cambio en procesos terapéuticos.

\section{Participantes}

Participaron 17 díadas (adolescentes - terapeutas) que habían realizado procesos terapéuticos en contextos naturales; $64,7 \%$ de los adolescentes de género femenino, con una media aproximada de edad de 16 años y diferentes diagnósticos; y 55,5\% de los terapeutas de género femenino y con una media de edad aproximada de 30 años. Se utilizó un muestreo teórico para la conformación de la muestra (Glaser \& Strauss, 2017/1967; Flick, 2012), con la intención de buscar variabilidad en el número de sesiones asistidas, enfoques teóricos y condición de finalización de la terapia (finalizada, abandono y en proceso), para explorar la percepción del proceso de cambio terapéutico de distintas experiencias y, así, dar profundidad conceptual al mismo (Tabla1).

\section{Recolección de información}

Se contactaron centros de atención en salud mental con atención a adolescentes y amplia cobertura social (centros universitarios de atención en salud mental abiertos a la comunidad, consultorios de atención primaria de salud gratuitos y un centro de salud privado). Todas las díadas terapeuta-adolescente que iniciaron terapia durante el proceso de recolección de información fueron invitadas a participar en el estudio. Los terapeutas, adolescentes y padres (en caso de minoría de edad del paciente) que consintieron y asintieron participar fueron contactados con un intervalo de tiempo, entre 2 y 11 meses luego de haber finalizado la terapia, según la disponibilidad de los pacientes y terapeutas.

Con cada participante de la diada se realizó una entrevista semiestructurada basada en un guion temático (Kvale, 2011; Larsen, Flesaker, \& Stege, 2008), que contiene una o dos preguntas introductorias amplias que propician la expresión espontánea de sus experiencias respecto al fenómeno investigado. Luego, se realizaron preguntas aclaratorias o más específicas para enriquecer la descripción de los relatos y profundizar las experiencias relatadas por cada sujeto (Flick, 2012) (Tabla 2). Los guiones se sometieron a una prueba piloto y, luego de las dos primeras entrevistas, se ajustaron de acuerdo con los resultados emergentes. Las entrevistas (audiograbadas y transcritas) fueron realizadas por asistentes de investigación ajenos a los centros de atención, con modalidad presencial, una duración aproximada de 60 minutos y de forma independiente con cada participante de la diada. 
Tabla 1. Descripción díadas participantes

\begin{tabular}{|c|c|c|c|c|c|c|c|c|}
\hline \multirow[b]{2}{*}{ ID } & \multicolumn{3}{|c|}{ Paciente } & \multicolumn{3}{|c|}{ Terapeuta } & \multirow{2}{*}{$\frac{N^{\circ}}{\text { Sesiones }}$} & \multirow{2}{*}{$\begin{array}{c}\text { Condición } \\
\text { Término }\end{array}$} \\
\hline & Edad & Género & $\begin{array}{c}\text { Diagnóstico } \\
\text { DSM* }^{*}\end{array}$ & Edad & Género & $\begin{array}{l}\text { Enfoque } \\
\text { teórico** }\end{array}$ & & \\
\hline 1 & 15 & F & TDP & 25 & F & Ecléctico & 6 & Abandona \\
\hline 2 & 17 & M & $\begin{array}{l}\text { Consumo de } \\
\text { sustancias }\end{array}$ & 26 & M & CE & 4 & Finaliza \\
\hline 3 & 19 & $F$ & - & 24 & M & $\begin{array}{l}\text { Psicodi- } \\
\text { námico }\end{array}$ & 130 & Continúa \\
\hline 4 & 12 & M & TDAH & 26 & M & CE & 64 & Finaliza \\
\hline 5 & 13 & $\mathrm{~F}$ & TCA & 24 & $\mathrm{~F}$ & Sistémico & 36 & Finaliza \\
\hline 6 & 14 & M & - & 24 & M & Sistémico & 20 & Finaliza \\
\hline 7 & 16 & $F$ & TDM Moderado & 56 & F & $\mathrm{CC}$ & 8 & Finaliza \\
\hline 8 & 17 & M & TDM Leve & 26 & $\mathrm{~F}$ & Ecléctico & 21 & Finaliza \\
\hline 9 & 13 & $\mathrm{~F}$ & TDM Leve & 26 & $\mathrm{~F}$ & Ecléctico & 10 & Finaliza \\
\hline 10 & 17 & $\mathrm{~F}$ & TDM Moderado & 56 & $\mathrm{~F}$ & $\mathrm{CC}$ & 11 & Finaliza \\
\hline 11 & 18 & $\mathrm{~F}$ & TDM Moderado & 56 & $\mathrm{~F}$ & $\mathrm{CC}$ & 6 & Abandona \\
\hline 12 & 18 & M & TDM Moderado & 37 & M & Ecléctico & 9 & Finaliza \\
\hline 13 & 16 & $\mathrm{~F}$ & TDM Severo & 31 & F & CCAC & 8 & Finaliza \\
\hline 14 & 15 & $\mathrm{~F}$ & TDM Moderado & 31 & F & CCAC & 8 & Finaliza \\
\hline 15 & 15 & M & TDM Moderado & 31 & $\mathrm{~F}$ & CCAC & 8 & Finaliza \\
\hline 16 & 17 & $\mathrm{~F}$ & TDM Moderado & 31 & $\mathrm{~F}$ & CCAC & 6 & Abandona \\
\hline 17 & 16 & $\mathrm{~F}$ & TDM Moderado & 31 & $\mathrm{~F}$ & CCAC & 5 & Abandona \\
\hline
\end{tabular}

*TDP=Trastorno del desarrollo de la personalidad; TDAH= Trastorno de Déficit Atencional con Hiperactividad; TCA= Trastorno de la conducta alimentaria, TDM= Trastorno de Depresión Mayor

${ }^{* *} \mathrm{CC}=$ Cognitivo-Conductual, $\mathrm{CCAC}=$ Cognitivo-Conductual asistida por Computador, $\mathrm{CE}=$ Constructivista Evolutivo.

Tabla 2. Guion temático

\begin{tabular}{|c|c|c|}
\hline Ejes temáticos & Preguntas Adolescentes & Preguntas Terapeutas \\
\hline Pregunta inicial & $\begin{array}{l}\text { "Cuéntame sobre cómo fue para ti } \\
\text { venir al psicólogo/a" }\end{array}$ & $\begin{array}{l}\text { "Cuéntame tu experiencia } \\
\text { respecto a la terapia con..." }\end{array}$ \\
\hline $\begin{array}{l}\text { a. Noción de problema } \\
\text { y expectativas }\end{array}$ & $\begin{array}{l}\text { ¿Por qué fuiste a terapia? ¿qué } \\
\text { expectativas tenías? ¿te gustaba o no } \\
\text { asistir? }\end{array}$ & $\begin{array}{l}\text { ¿Por qué vino el/la adolescente? } \\
\text { ¿tenía alguna expectativa? } \\
\text { ¿pensabas que le podrías ayudar } \\
\text { en algo? }\end{array}$ \\
\hline $\begin{array}{l}\text { b. Relación } \\
\text { terapéutica }\end{array}$ & $\begin{array}{l}\text { ¿Cómo era la relación con tu } \\
\text { psicólogo? ¿cómo te sentías con } \\
\text { él/ella al principio de la terapia y } \\
\text { después? }\end{array}$ & $\begin{array}{l}\text { ¿Qué actitud tenía al venir? ¿cómo } \\
\text { te sentías con él/ella? ¿Cómo se } \\
\text { fue dando la relación? }\end{array}$ \\
\hline $\begin{array}{l}\text { c. Caracterización } \\
\text { del proceso y } \\
\text { atribuciones del } \\
\text { cambio }\end{array}$ & $\begin{array}{l}\text { ¿Qué hacían en las sesiones? ¿cómo } \\
\text { te sentías? ¿Hubo algún momento } \\
\text { significativo positivo o negativo que } \\
\text { recuerdes? }\end{array}$ & $\begin{array}{l}\text { ¿Cómo eran las sesiones, qué } \\
\text { hacían? ¿tenían alguna rutina? }\end{array}$ \\
\hline $\begin{array}{l}\text { d. Cambio / no cambio: } \\
\text { atribuciones }\end{array}$ & $\begin{array}{l}\text { ¿Te ayudó en algo ir a terapia? } \\
\text { ¿notaste algún cambio en ti? ¿por qué } \\
\text { y cómo crees que la terapia te sirvió? }\end{array}$ & $\begin{array}{l}\text { ¿Notaste algún cambio en él/ } \\
\text { ella? ¿cómo te diste cuenta de } \\
\text { ese cambio? ¿en qué crees que le } \\
\text { sirvió la terapia? }\end{array}$ \\
\hline
\end{tabular}


Pág 113

El fenómeno central que cruza toda la experiencia terapéutica es el encuentro con otro (Figura 1). A este encuentro los adolescentes llegan sin expectativas y motivaciones específicas, más bien de forma pasiva, resignados por el mandato de los padres, colegios o médicos. Existe cierta curiosidad respecto a la experiencia, pero no se manifiestan expectativas explícitas de resultados o logros. Ellos solo tienen expectativas acerca de la persona del terapeuta y de la relación, en cuanto a que sea agradable y no sea hostil ni enjuiciadora.
La presente investigación se enmarca en el proyecto FONDECYT N¹141179, "Experiencias de Éxito y Fracaso en Psicoterapia - Construcción de un Modelo Comprehensivo Multidimensional". Este proyecto fue aprobado por el Comité de Ética de la Escuela de Psicología de la Pontificia Universidad Católica de Chile. La participación en el estudio fue voluntaria e informada, los participantes firmaron los respectivos consentimiento y asentimiento; y la información recolectada fue manejada confidencialmente.

\section{Análisis de la información}

La información obtenida mediante las entrevistas fue analizada según los procedimientos de la Teoría Fundamentada (Charmaz, 2006; Corbin \& Strauss, 1990). En primer lugar, codificadores expertos realizaron una codificación abierta de los datos. Este proceso consiste en fragmentar, examinar y comparar los datos, para desarrollar conceptos y categorías de carácter emergente, los cuales fueron organizados en esquemas de clasificación jerárquicos para el desarrollo de categorías descriptivas. Posteriormente, se realizó la codificación axial, es decir, un análisis relacional e interpretativo entre las categorías descriptivas (Corbin \& Strauss, 1990; Flick, 2012), que dio como resultado una organización de esquemas relacionales. Los procesos de recolección y análisis de datos estuvieron articulados, lo que aseguró una producción de datos guiada por los criterios de saturación del campo de estudio y de las categorías emergentes.

El análisis de la información fue asistido por el programa computacional ATLAS. TI v7, diseñado para el manejo y procesamiento de datos textuales. Para asegurar la calidad de los resultados, se empleó la estrategia de triangulación entre codificadores (Krause, 1995), y todos los investigadores participaron en los procesos de análisis cualitativo de los datos, de manera que la identificación e interpretación de las categorías generadas se validara a través del acuerdo intersubjetivo. Asimismo, se aplicó el criterio de descripción densa (Ponterotto, 2006), es decir, se buscó comprender e interpretar de manera contextualizada cada una de las entrevistas, para describir plausiblemente cada uno de los elementos que configuran los relatos.

\section{Resultados}

La presentación de los resultados se organizó en torno a dos fenómenos relacionales emanados del discurso de los actores del proceso terapéutico; el primero se denominó "Encuentro con otro: plataforma segura y sustrato relacional del cambio" y se refiere a aspectos de la interacción establecida en el espacio terapéutico, y, el segundo "Cambio: empoderamiento y Valoración de sí", se refiere a la comprensión de los procesos o mecanismos asociados a la generación del cambio terapéutico.

\section{Encuentro con otro: plataforma segura y sustrato relacional del cambio}

El fenómeno central que cruza toda la experiencia terapéutica es el encuentro con otro (Figura 1). A este encuentro los adolescentes llegan sin expectativas y motivaciones específicas, más bien de forma pasiva, resignados por el mandato de los padres, colegios o médicos. Existe cierta curiosidad respecto a la experiencia, pero no se manifiestan expectativas explícitas de resultados o logros. Ellos solo tienen expectativas acerca de la persona del terapeuta y de la relación, en cuanto a que sea agradable y no sea hostil ni enjuiciadora. Así lo expresa uno de los participantes en el estudio: 
Si el/la joven se siente cómodo y en sintonía con el terapeuta permanece en el proceso terapéutico, así lo manifiesta uno ellos: "Es que no me costó llegar a ella como persona, no, no fue como que yo tuviera que trabajar una, o sea, traspasar una barrera profesional-paciente (...) no sentía distante la relación, a eso me refiero" (Adolescente 14). Luego, paulatinamente, se irá abriendo a la relación y a la conexión con el/la terapeuta.
La verdad es que no me gusta conversar con la gente que no conozco, no me gusta. Entonces como que fui porque tenía que ir, porque tenía que conversarle a esa vieja [...] y yo me decía: 'ay! ¿por qué tengo que ir?' y '¿quién es ella? ¿y si es pesada [desagradable]?', yo tenía mucha lata [pereza]. Y yo como que me imaginaba a mis profes, así, como a mi profe [profesora] de filosofía que era súper pesada [desagradable], yo me la imaginaba como a ella, así. Y no, y como que fui y en realidad la psicóloga es como súper simpática, entonces, yo iba como con el presupuesto [prejuicio] de que ella iba a ser muy pesada [desagradable] y, pero no, tal vez fue como una grata sorpresa. (Adolescente 10).

El género del terapeuta parece tener incidencia en la disposición de los adolescentes y en el establecimiento del clima de confianza, especialmente para los adolescentes de género femenino, quienes prefieren terapeutas de su mismo género, según lo dice una de ellas: "Para mi suerte [la terapeuta] era una mujer, porque yo me siento muy incómoda con los hombres, psicólogos y doctores, me siento muy incómoda, pero que fuera una mujer me ayudó bastante a poder confiar en la persona" (Adolescente 14). Asimismo, tener intereses en común, facilita el establecimiento paulatino de la confianza del adolescente en la terapia, como dice este terapeuta:

Yo igual tenía cosas en común con [el adolescente], y yo creo que eso fue la clave (...) yo también me conectaba mucho más con él, y yo creo que esa fue la clave para que él pudiese como, por ejemplo, ser bastante receptivo con, con la psicoterapia. (Terapeuta 5)

Los terapeutas, según la percepción de los adolescentes, tienen disposición y motivación para cumplir su rol de ayuda y están comprometidos con hacer un buen trabajo, así lo expresa uno de ellos: "Estaba dispuesto a ayudarme, no como, ejemplo, los doctores que te dicen 'ya, esto tómate'...no, él tenía la disposición de, de ayudar, y pensaba y pensaba para ver cómo me ayudaba" (Adolescente 8). A su vez, la experiencia relacional de los padres con el terapeuta, sus actitudes y opiniones acerca de este, son aspectos que afectan tanto a los adolescentes como al terapeuta, según lo manifiesta uno de los profesionales: "Esta mamá era bien empática y la acompañó, y validó el espacio terapéutico" (Terapeuta 13).

Si el/la joven se siente cómodo y en sintonía con el terapeuta permanece en el proceso terapéutico, así lo manifiesta uno ellos: "Es que no me costó llegar a ella como persona, no, no fue como que yo tuviera que trabajar una, o sea, traspasar una barrera profesional-paciente (...) no sentía distante la relación, a eso me refiero" (Adolescente 14). Luego, paulatinamente, se irá abriendo a la relación y a la conexión con el/la terapeuta, como señala un terapeuta:

Al principio a mí me costó harto establecer una relación terapéutica de confianza. Porque ella siempre estaba a la defensiva, pensaba que todo lo que yo le hacía era como que le iba a decir al papá. Hasta que se dio cuenta que no, y al final ella pudo sentirse cómoda conmigo y ahí empezamos a trabajar harto (Terapeuta 4).

En el mismo sentido, si el adolescente no se siente cómodo y tranquilo, ni en sintonía con el terapeuta, no continua con el proceso, tal como lo reportó una de las jóvenes que no concluyó su terapia: "[Mi mamá] Me dijo que no le vio [a la terapeuta] mucho interés, que era solamente por el proyecto que estaban haciendo no más, como que no le vio mucho interés en mí, entonces... eso es lo que yo sentí" (Adolescente 16). 
Así, la psicoterapia se irá construyendo como un espacio propio y protegido, donde el/la adolescente puede conversar libremente sin ser juzgado y en confidencialidad, como lo afirman dos de ellos: "Era cariñosa, da confianza, uno le decía algo y era por seguro que no le iba a decir a nadie" (Adolescente 13); "Como que alguien me escuchara y alguien que yo no conociera que yo pudiera como confiar en él” (Adolescente 6).

En este espacio, el/la adolescente plantea que se siente en una relación diferente a las que sostiene habitualmente con otros adultos y que el/la terapeuta, aunque es un adulto, se percibe como un igual que valida sus experiencias, opiniones y sentimientos, con genuino interés de conocerlo/a. A su vez, el/la adolescente es capaz de reconocer y sentir que el/la terapeuta está en un rol profesional, tiene experiencia y conocimiento y valida la asimetría en lo profesional, pero en una relación de cercanía afectiva que genera sentimientos reales y que permite el proceso de cambio, así lo afirma uno de los terapeutas:

"Creo que ella sintió que esto es un espacio para ella y que ella podía hablar de sus cosas, y que no se le juzgaba, en que además las cosas que ella decía quedaban dentro de ese vínculo, y estableció una buena confianza. También, cuando fue necesario abrirle el espacio terapéutico hacia el resto de la familia [incorporar familiares a las sesiones], se hizo y que ella vio resultados, y yo creo que eso la ayudó mucho" (Terapeuta 6).

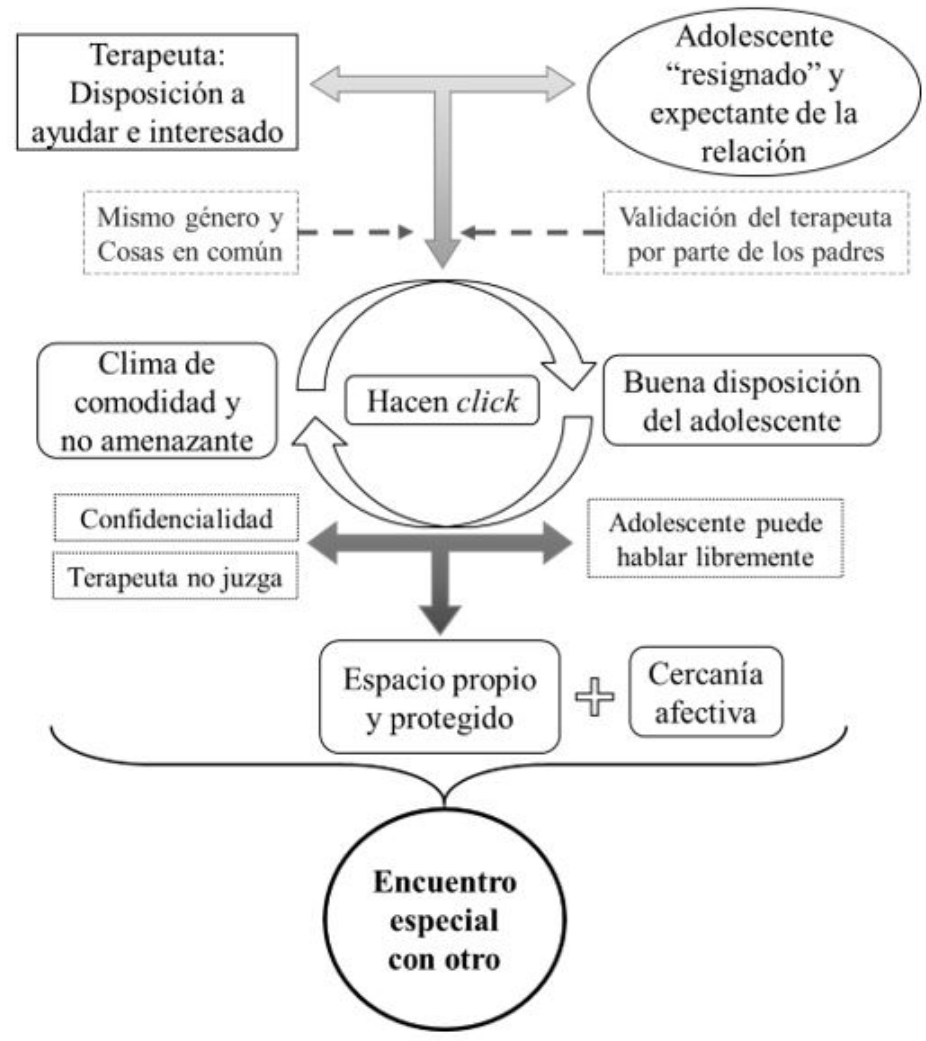

Figura 1. Encuentro con otro: plataforma segura y sustrato relacional del cambio. Fuente propia

En síntesis, este encuentro especial con el otro empieza incluso antes de iniciar la psicoterapia, con las expectativas del adolescente enfocadas en la persona del terapeuta y en la relación con este; que son indicios de la importancia de este encuentro para el/ 
Pág 116

El trabajo y el cambio terapéutico se presentan de forma imbricada e inseparable de la relación terapéutica (Figura 2). Las experiencias surgidas en la interacción psicoterapéutica activan o desactivan la disposición a trabajar en la construcción de cambios. la adolescente. Al encontrarse con un terapeuta dispuesto a ayudar y escuchar, que expresa un interés genuino hacia él, su reticencia inicial se va transformando en una disposición de apertura hacia la relación y el espacio terapéutico, y se crea un clima de confianza. En este momento, el adolescente se siente en sintonía con el terapeuta y, gracias a sentirse protegido por la confidencialidad y actitud comprensiva de este/ esta, siente que puede hablar libremente y construir un espacio protegido especial para él/ella. Esto, más la construcción de una cercanía afectiva, permiten el encuentro con el otro, el desarrollo de una relación diferente a cualquier otra que tiene en su vida (con padres o pares), en un espacio único. Esta relación, más la validación y confianza en el terapeuta, brindan el sustrato relacional para hacer posible la construcción del cambio terapéutico.

\section{Cambio: Empoderamiento y validación de sí}

El trabajo y el cambio terapéutico se presentan de forma imbricada e inseparable de la relación terapéutica (Figura 2). Las experiencias surgidas en la interacción psicoterapéutica activan o desactivan la disposición a trabajar en la construcción de cambios.

Desde el inicio, el terapeuta comunica a través de acciones simples, pero intencionadas, que el espacio psicoterapéutico es personalizado y co-construido según las características y necesidades del/la paciente. Esto se traduce en una estructura de sesión flexible, que considera y valora la opinión del adolescente, y se adapta a sus necesidades (horarios, temáticas a tratar, actividades). De forma contraria, cuando los adolescentes perciben que las sesiones están organizadas sólo en función de criterios del terapeuta (temáticas y actividades prefijadas), sienten que estas intervenciones no respetan su individualidad y dejan sus necesidades en segundo plano. Esto se percibe en el testimonio de una adolescente que abandona la terapia:

Ella era simpática, pero el problema era que solamente me decía 'completa esto, completa esto' y no me preguntaba 'oye, ¿cómo estás? ¿Cómo te ha ido? nada, solamente los programas y 'nos vemos hasta la otra sesión y chao'. Entonces no, siento que no aportó casi nada (Adolescente 16).

En esta interacción el/la terapeuta muestra completa disposición a escuchar, consignar y registrar las experiencias del otro presente, el/la adolescente aumenta su involucración y participación en el proceso: reconoce que debe hablar de sí y de sus relaciones familiares y sociales, preguntar y opinar. Así lo expresa uno de los profesionales entrevistados:

De hecho, había momentos en que nos tomaba más tiempo [las sesiones], tratábamos de resolver cosas como más contingentes para ella, que no eran propiamente de este plan cognitivo-conductual de depresión, sino que cosas más en relación con.... me contaba algo de sus temas amorosos (Terapeuta 15).

Cuando el/la adolescente tiene dificultades para comunicar sus experiencias, el terapeuta toma un rol más directivo, busca áreas y actividades específicas de su interés, aunque parezcan irrelevantes al motivo de consulta (tocar guitarra, dibujar, juegos de video, libros, entre otros). Tal como se expresa en el siguiente testimonio: 
Pág 117

Los/as adolescentes destacan la capacidad del terapeuta de recordar sus experiencias y emociones de sesiones anteriores, lo que es interpretado por ellos/ as como una expresión de interés genuino y valoración por su persona.
Le pedí que trajera cosas que le gustan, y me trajo la guitarra, estuvimos como dibujando y poniendo cosas que iban saliendo, y ahí iba intentando que eso lo fuera aplicando [reflexionando] con un poquito de criterio de realidad [yo le decía] 'oye mira esto, ¿qué piensas tú de esto? (Terapeuta 3).

Los/as adolescentes destacan la capacidad del terapeuta de recordar sus experiencias y emociones de sesiones anteriores, lo que es interpretado por ellos/as como una expresión de interés genuino y valoración por su persona. Así se ve expresado en esta respuesta:

Lo que cambió [mi percepción] de ella [la terapeuta], era que igual se acordaba de las cosas que yo le decía y yo no. Yo me imaginaba que ella tiene lleno de pacientes, que anda pendiente de miles de personas, entonces me sorprendía que se acordara de cosas que uno le decía, siendo que ella atiende a pacientes casi toda la semana (Adolescente 7).

Así, el/la adolescente registra que el/la terapeuta lo tiene en mente, lo que estimula aún más su proceso de apertura y compromiso con la psicoterapia. Ahora es capaz de aceptar nuevas miradas de problemas o situaciones ofrecidas por el terapeuta, y cuestionar y flexibilizar opiniones y actitudes, a través de la reflexión y análisis de nuevas perspectivas de sí, los otros (padres, amigos, hermanos) y de los problemas, según se expresa en este testimonio:

Lo que hizo [el terapeuta] era algo que necesitaba, o sea, a mí me sorprendió, yo creí que me iba como a escuchar y decir como 'todo va a estar bien'. Pero en realidad me dijo como 'no, tienes que hacer esto', me dio como alternativas, cosas que a mí no se me ocurren (Adolescente 9).

La capacidad de tomar perspectiva se complementa con acciones del terapeuta destinadas a empoderar al adolescente; se le desafía y estimula a buscar sus propias alternativas, tomar decisiones y desarrollar habilidades para abordar sus problemas. Así lo expresa esta adolescente:

[la terapeuta] siempre me generó como harta confianza, y siempre fue súper sincera, pero, en comparación a otras personas, nunca me dijo: 'no, es que tú estás mal en esto, en esto, en esto'. Yo misma me di cuenta de las cosas en que yo estaba mal, yo (...) nunca me dijo lo que yo tenía mal, pero me hizo darme cuenta a mí (Adolescente 1).

Por su parte, la terapeuta dice:

Estimulé mucho el sistema del self en él, lo más que pude, en la lógica de que él era muy, muy lento [pasivo]. Entonces creo yo que el tratamiento, ¿en qué le aportó? en la posibilidad de que él pudiera empoderarse, que él pudiera tomar las riendas de su vida y pudiera tomar algunas decisiones que podían resultarle mejor (Terapeuta 12). 
Pág 118

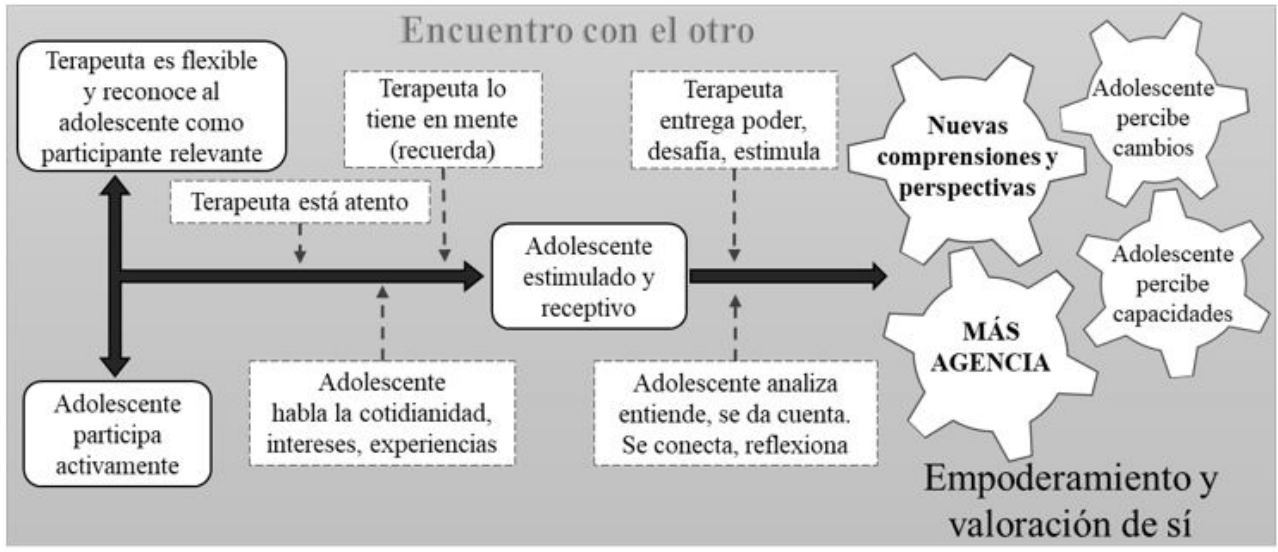

Figura 2: Cambio: empoderamiento y valoración sí mismo. Fuente: propia

Una psicoterapia con adolescentes generadora de cambios proporciona una experiencia diferente, de seguridad, validación y empoderamiento, a través de un profesional calificado, que construye un encuentro relacional que ofrece protección y aceptación y, al mismo tiempo, realiza intervenciones que estimulan y desafían las estructuras de pensamiento y modos de relacionarse del adolescente consigo y con los demás, en busca de configurar una identidad positiva.
De esta forma, el adolescente va descubriendo y desarrollando nuevas capacidades para elaborar y responder a las situaciones que se le presentan; gana agencia sobre sí mismo, al tiempo que percibe cambios y mejoras, y refuerza su sentimiento de poder sobre sí mismo y sus circunstancias (empoderamiento), lo que aporta a su propia valoración. Por esto, el cambio no es consignado solo como la resolución específica de los problemas que lo llevaron al psicólogo, sino también como la revalidación de su persona, la adquisición de herramientas, confianza y mayor valoración de sí mismo, así como la toma de conciencia sobre sus particularidades y su manera de ser.

\section{Discusión}

Los resultados de este estudio muestran dos grandes mecanismos en el proceso de cambio psicoterapéutico en los adolescentes desde la perspectiva de ellos mismos y sus terapeutas. Primero, el adolescente vive una experiencia relacional de confianza, aceptación y validación de sí como sujeto de interés y valor para otro. Esta experiencia relacional es el sustrato esencial para que se presente el segundo mecanismo de cambio, que consiste en lograr el empoderamiento y la valoración de sí mismo en el espacio psicoterapéutico. Esta experiencia de sí le facilita al adolescente poder mirarse y mirar a otros desde nuevas perspectivas, al igual que redescubrir potencialidades y nuevas formas de enfrentarse a las dificultades, lo cual refuerza su identidad.

En un sentido genérico, los resultados del estudio indican que el cambio en psicoterapia con adolescentes se relaciona íntimamente con el logro de las tareas del desarrollo propias de la edad, tales como el fortalecimiento de su identidad. Esto es coherente con otros trabajos, provenientes de distintos países y contextos culturales (Almonte, 2019; Capella et al., 2017; Kazdin, 2004), en los que se plantea que el principal objetivo de la psicoterapia con niños y adolescentes es reinstalarlos en el curso normal del desarrollo; dado que, según la psicopatología evolutiva las desviaciones de este curso son el principal factor de desajuste o disfunción. En este sentido, una psicoterapia con adolescentes generadora de cambios proporciona una experiencia diferente, de seguridad, validación y empoderamiento, a través de un profesional calificado, que construye un encuentro relacional que ofrece protección y aceptación y, al mismo tiempo, realiza intervenciones que estimulan y desafían las estructuras de pensamiento y modos de relacionarse del adolescente consigo y con los demás, en busca de configurar una identidad positiva. 
Pág 119

Esta interacción-intervención del adolescente con el/ la terapeuta evoca la interrelación función materna/paterna y construcción de la mente infantil (Fonagy, Gergely, \& Jurist, 2018) en la que, a través de una función de contención y sintonía afectiva, el otro va reconociendo su self y el de los otros, se podría decir, desarrolla su capacidad de mentalización o función reflexiva.
En el modelo de cambio en psicoterapia con adolescentes, que resultó del presente estudio, la figura del terapeuta toma diversas posiciones: de un lado, asume un rol activo en la gestación y mantenimiento del espacio relacional seguro; en este proceso pone en juego sus habilidades y destrezas técnicas específicas para lograr instalar y mantener un espacio de estas características. De otro lado, asume un rol pasivo -se deja guiar- por el/la adolescente, que es el protagonista activo y guionista de la psicoterapia, que propone temas, sus necesidades e intereses; presenta sus relatos y sus silencios; sus ritmos y sus objetivos terapéuticos. En este papel, el terapeuta ajusta el trabajo a las características del adolescente, a su contexto familiar y social.

Según Altimir, Capella, Núñez, Abarzua y Krause (2017) una relación psicoterapéutica tiene una dimensión asimétrica en lo profesional (técnica y de función), y simétrica en lo relacional, referida al intercambio afectivo existencial, similar al concepto de relación real (Gelso, \& Kline, 2019). Esta dimensión relacional está presente en todos los procesos psicoterapéuticos, sin embargo, dadas las particularidades de los adolescentes (Bhola \& Kapur, 2013; Everall \& Paulson, 2002; Fernández et al.2016), es de vital importancia como factor de cambio en la psicoterapia con este grupo etario. Es posible pensar que, dada la necesidad de configuración de la identidad y los procesos que eso conlleva, los adolescentes son más sensibles que los adultos a los estilos relacionales seguros o inseguros. Esto permite entender la hipersensibilidad de los adolescentes a los menores signos de distanciamiento por parte de los terapeutas, lo que les genera desconfianza en el espacio terapéutico. En esta línea, Morán et al. (2019), en un estudio con terapeutas de adolescentes acerca de rupturas de la alianza terapéutica (Safran \& Muran, 2006), plantean que éstas se relacionan con la baja consideración del terapeuta de la experiencia inmediata del adolescente (el terapeuta llega con su propia agenda a la sesión) y con una alta reactividad y sensibilidad a los errores del terapeuta por parte del adolescente. Así, los terapeutas deben estar en constante alerta a los efectos interaccionales de sus intervenciones. Lo anterior, valida una vez más, que la relación terapéutica es el factor crítico que permite anidar o abortar cualquier intervención con adolescentes.

Ahora bien, en cuanto al cambio psicoterapéutico en los adolescentes, los resultados destacan que el desarrollo de nuevas perspectivas de sí, de los otros (padres, amigos, hermanos) y de los problemas es facilitado por la interacción confiada y las intervenciones terapéuticas. De manera progresiva, durante la psicoterapia el/la joven participa, conversa y se va mostrando; el terapeuta a su vez, se interesa en descubrir la esencia del adolescente y su forma de ver las cosas, para luego invitarlo a entender y analizar la opinión o actitud de los otros. Esta interacción-intervención del adolescente con el/la terapeuta evoca la interrelación función materna/paterna y construcción de la mente infantil (Fonagy, Gergely, \& Jurist, 2018) en la que, a través de una función de contención y sintonía afectiva, el otro va reconociendo su self y el de los otros, se podría decir, desarrolla su capacidad de mentalización o función reflexiva. Este desarrollo de la función reflexiva se relaciona con el sentimiento de propiedad de las acciones que uno lleva a cabo, lo que es básico para lograr el sentido de agencia de sí mismo (Stern, 1985).

También, los resultados del estudio destacan ciertas intervenciones a través del diálogo en sesión: conversación abierta, narrativa de vida cotidiana por parte del adolescente, en un contexto de ausencia de crítica. Estos hallazgos son concordantes con un estudio sobre acciones comunicativas en las sesiones terapéuticas de terapias exitosas con adolescentes, donde las formas verbales orientadas a explorar y el preguntar son las de mayor proporción (Krause et al., 2015). 
La identificación de los fenómenos comprensivos relacionados con los procesos de cambio psicoterapéutico representa un gran desafío para los terapeutas, ya que le demandan una identificación y empatía con el/la adolescente, que les permitan entenderlo y hacer de espejo y, a la vez, cumplir la función de cuidado, contención y responsabilidad técnica profesional. Sin duda esta doble función requiere de formación especializada y supervisión con pares.

El modelo relacional comprensivo del cambio psicoterapéutico en adolescentes presentado aquí, es genérico, vale decir, transversal a diferentes enfoques psicoterapéuticos, pero los mecanismos de cambio observados son específicos a la psicoterapia con adolescentes, y tienen relación con su etapa evolutiva particular.

Este estudio presenta en su mayoría díadas terapéuticas que lograron terminar el proceso, por lo que sería relevante enriquecer estos hallazgos con díadas que lo hayan interrumpido, o que explícitamente se hayan sentido insatisfechos con la terapia. Así mismo, explorar los cambios y mecanismos de cambio en relación con motivos de consulta o psicopatologías específicas, por ejemplo, trastornos externalizantes versus internalizantes, o trastornos del desarrollo, constituyen desafíos para futuras investigaciones.

Este trabajo fue financiado por ANID - Iniciativa Científica Milenio / Instituto Milenio para la Investigación en Depresión y Personalidad-MIDAP ICS13_005.

\section{Referencias}

Almonte, C. (2019) Características generales de la psicopatología infantil y de la adolescencia. En C., Almonte, \& M.E., Montt. (eds.). Psicopatología Infantil y de la Adolescencia. Tercera Edición (pp. 19 - 28).

Altimir, C., Capella, C., Núñez, L., Abarzua, M., \& Krause, M. (2017). Meeting in difference: Revisiting the therapeutic relationship based on patients- and therapistsexperiences in several clinical contexts. Journal of Clinical Psychology, 73(11), 1510-1522. https://doi.org/10.1002/jclp.22525

Benjet, C. (2009). La salud mental de la niñez y la adolescencia en América Latina y el Caribe. En J., Rodriguez, R., Kohn, \& S., Aguliar-Gaxiola. (Eds) Epidemiología de los trastornos mentales en América Latina y el Caribe, (pp. 234-242) Washington, D.C.: Organización Panamericana de la Salud.

Bhola, P., \& Kapur, M. (2013). The development and role of the therapeutic alliance in supportive psychotherapy with adolescents. Psychological Studies, 58(3), 207215. https://doi.org/10.1007/s12646-013-0191-0

Binder, P. E., Holgersen, H., \& Nielsen, G. H. (2008). Establishing a bond that works: A qualitative study of how psychotherapists make contact with adolescent patients. European Journal of psychotherapy and counselling, 10(1), 55-69. https:// doi.org/10.1080/13642530701869730

Bordin, E. S. (1976). The generalizability of the psychoanalytic concept of the working Alliance. Psychotherapy: Theory, Research, and Practice, 16, 252 -260.

Capella, C., Soza, J., García, G., Sepúlveda, G., Quiroga, F., González, I., ... Vergara, P. (2017). Cambio psicoterapéutico en niños/as y adolescentes desde el enfoque constructivista evolutivo: evaluación de un caso. Revista Argentina de Clínica Psicológica, 26(1), 125-136.

Charmaz, K. (2006). Constructing grounded theory. A practical guide through qualitative analysis. Los Angeles, CA: Sage.

Corbin, J. M., \& Strauss, A. (1990). Grounded theory research: Procedures, canons, and evaluative criteria. Qualitative sociology, 13(1), 3-21. 
Corbella, S., \& Botella, L. (2003). La alianza terapéutica: historia, investigación y evaluación. Anales de Psicología, 19(2), 205-221.

Creed, T. A., \& Kendall, P. C. (2005). Therapist Alliance-Building Behavior within a Cognitive-Behavioral Treatment for Anxiety in Youth. Journal of Consulting and Clinical Psychology, 73(3), 498-505. https://doi.org/10.1037/0022-006X.73.3.498

Daly, A. M., Llewelyn, S., McDougall, E., \& Chanen, A. M. (2010). Rupture resolution in cognitive analytic therapy for adolescents with borderline personality disorder. Psychology and Psychotherapy: Theory, Research and Practice, 83(3), 273-288. https://doi.org/10.1348/147608309X481036

Diamond, G., Siqueland, L., \& Diamond, G.M. (2003). Attachment-Based Family Therapy for Depressed Adolescents: Programmatic Treatment Development. Clin Child Fam Psychol Rev, 6(2), 107-127. https://doi.org/10.1023/A:1023782510786

DiGiuseppe, R., Linscott, J., \& Jilton R (1996) Developing the therapeutic alliance in child-adolescent psychotherapy. Applied and Preventive Psychology, 5(2), 85-100. https://doi.org/10.1016/S0962-1849(96)80002-3

Everall, R. D., \& Paulson, B. L. (2002). The therapeutic alliance: adolescent perspectives. Counselling and Psychotherapy Research, 2(2), 78-87. https://doi.org/10.10 $\underline{80 / 14733140212331384857}$

Fernández, O. (2019). El trabajo con los padres en la terapia de niños y adolescentes. En C., Almonte, \& M.E., Montt. (eds.). Psicopatología Infantil y de la Adolescencia. Tercera Edición (pp.762 - 769).

Fernández, 0., Herrera-Salinas, P., \& Escobar-Martínez, M. J. (2016). Adolescentes en psicoterapia: Su representación de la relación terapéutica. Revista Latinoamericana de Ciencias Sociales, Niñez y Juventud, 14(1), 559-575.

Fernández, 0., Krause, M., \& Pérez, J. C. (2016). Therapeutic alliance in the initial phase of psychotherapy with adolescents: different perspectives and their association with therapeutic outcomes. Research in Psychotherapy: Psychopathology, Process and Outcome, 19(1), 4-18. https://doi.org/10.4081/ripppo.2016.180

Fernández, O., Pérez, J. C., \& Krause, M. (2019). The relation between the therapeutic alliance and communicative intentions in therapeutic interaction during the initial phase of adolescent therapy. Research in Psychotherapy: Psychopathology, Process and Outcome, 22(2), 189-198. https://doi.org/10.4081/ripppo.2019.356

Flick, U. (2012). Introducción a la investigación cualitativa. Madrid Ediciones Morata. Tercera reimpresión

Fonagy, P., Gergely, G., \& Jurist, E. L. (Eds.). (2018). Affect regulation, mentalization and the development of the self. London: Routledge.

García, R. (2019). La salud mental y perspectivas clínicas en la infancia y la adolescencia. En C., Almonte, \& M.E., Montt. (eds.). Psicopatología Infantil y de la Adolescencia. Tercera Edición (pp.243 - 249). Chile: Ed. Mediterráneo.

Gelso, C. J., \& Kline, K. V. (2019). The sister concepts of the working alliance and the real relationship: on their development, rupture, and repair. Research in Psychotherapy: Psychopathology, Process and Outcome, 22(2), 142-149. https://doi. org/10.4081/ripppo.2019

Glaser, B. G., \& Strauss, A. L. (2017). Discovery of grounded theory: Strategies for qualitative research. Third edition (First edition 1967). New York: Routledge.

Goodyer, I. M., Tsancheva, S., Byford, S., Dubicka, B., Hill, J., Kelvin, R., ... Fonagy, P. (2011). Improving mood with psychoanalytic and cognitive therapies (IMPACT): a pragmatic effectiveness superiority trial to investigate whether specialised psychological treatment reduces the risk for relapse in adolescents with moderate to severe unipolar depression: study protocol for a randomised controlled trial. Trials, 12(1), 175 - 187. https://doi.org/10.1186/1745-6215-12-175 
Hoffart, A., Borge, F- M., Sexton, H., Clark, D., \& Wampold, B. (2012). Psychotherapy for social phobia: How do alliance and cognitive process interact to produce outcome? Psychotherapy Research, 22(1), 82 - 94. https://doi.org/10.1080/10503307.2011. $\underline{626806}$

Horvath, A. O. (2001). The therapeutic alliance: Concepts, research, and training. Australian Psychologist, 36(2), 170-176. https://doi.org/10.1080/00050060108 259650

Karver, M., Shirk, S., Handelsman, J. B., Fields, S., Crisp, H., Gudmundsen, G., \& McMakin, D. (2008). Relationship processes in youth psychotherapy: Measuring alliance, alliance-building behaviors, and client involvement. Journal of Emotional and Behavioral Disorders, 16(1), 15-28. https://doi.org/10.1177\%2F1063426607312536

Kazdin, A (2004). Psychotherapy for Children and Adolescents. En M., Lambert (Ed.), Bergin and Garfield's Handbook of Psychotherapy and Behavior Change. New York: Wiley \& Sons.

Kazdin, A. E. (2007). Mediators and mechanisms of change in psychotherapy research. Annual Review of Clinical Psychology, 3(1), 1-27. https://doi.org/10.1146/ annurev.clinpsy.3.022806.091432

Kazdin, A. E. (2009). Understanding how and why psychotherapy leads to change. Psychotherapy research, 19(4-5), 418-428. https://doi. org/10.1080/10503300802448899

Kessler, R. C., Angermeyer, M., Anthony, J. C., De Graaf, R. O. N., Demyttenaere, K., Gasquet, I., ... Kawakami, N. (2007). Lifetime prevalence and age-of-onset distributions of mental disorders in the World Health Organization's World Mental Health Survey Initiative. World psychiatry, 6(3), 168.

Kessler, R. C., Berglund, P., Demler, O., Jin, R., Merikangas, K. R., \& Walters, E. E. (2005). Lifetime prevalence and age-of-onset distributions of DSM-IV disorders in the National Comorbidity Survey Replication. Archives of General Psychiatry, 62(6), 593-602. https://doi.org/10.1001/archpsyc.62.6.593

Krause, M. (1995). La investigación cualitativa: Un campo de posibilidades y desafíos. Revista Temas de Educación, 7(7), 19-40.

Krause, M., Fernández, O. M., \& Bräutigam, 0. (2015). Ergebnisqualität von psychotherapeutischen Prozessen mit Jugendlichen. Psychotherapeut, 60(5), 419-425. https://doi.org/10.1007/s00278-015-0037-4

Kvale, S. (2011). Las entrevistas en investigación cualitativa. Madrid: Ediciones Morata.

Larsen, D., Flesaker, K., \& Stege, R. (2008). Qualitative interviewing using Interpersonal Process Recall: Investigating internal experiences during professional-client conversation. International Journal of Qualitative Method, 7(1), 18-37. https://doi. org/10.1177/160940690800700102

Levisky, D. L. (1999). Adolescencia: reflexiones psicoanalíticas. Buenos Aires: Editorial Lumen.

Manríquez, H., Molina, H., \& Zubarew, T. (2003). Promoción y prevención de salud en la adolescencia. Adolescencia, promoción, prevención y atención de salud. Santiago: Ediciones Universidad Católica de Chile.

Marchesi, Á., Palacios, J., \& Coll, C. (2017). Desarrollo psicológico y educación. Madrid: Alianza Editorial.

Martin, D.J., Garske, J.P., \& Davies, M.K. (2000). Relation of therapeutic alliance with outcome and other variables: A meta-analytic review. Journal of Consulting and Clinical Psychology, 68(3), 438-450. https://doi.org/10.1037/0022-006X.68.3.438

Midgley, N., Capella, C., Goodman, G., Lis, A., Noom, M., Tishby, O., \& Weitkamp, K. (2018). Introduction to the special section on child and adolescent psychotherapy research. Psychotherapy Research, 28(1), 1-2. https://doi.org/10.1080/1050330 $\underline{7.2017 .1380864}$ 
Morán, J., Díaz, M. F., Martínez, C., Varas, C., \& Sepúlveda, R. P. (2019). The subjective experience of psychotherapists during moments of rupture in psychotherapy with adolescents. Research in Psychotherapy: Psychopathology, Process and Outcome, 22(1), 34-44. https://doi.org/10.4081/ripppo.2019.346

Páramo, M. (2011). Psicología Clínica de Niños y Adolescentes Psicoterapia para Adolescentes y Perfil del Psicólogo: Análisis de contenido de discursos grupales. Revista Argentina de Clínica Psicológica, 20(2), 133-142. Recuperado de https:// www.redalyc.org/articulo.oa? id $=2819 / 281922823004$

Ponterotto, J. G. (2006). Brief note on the origins, evolution, and meaning of the qualitative research concept "thick description". The Qualitative Report, 11(3), 538-549.

Russell, R. (2008). Child and Adolescent psychotherapy: Introduction to the special section. Psychotherapy Research, 18(1), 1-4. https://doi.org/10.1080/1050330070 1725090

Russell, R., Shirk, S., \& Jungbluth, N. (2008). First-session pathways to the working alliance in cognitive-behavioral therapy for adolescent depression. Psychotherapy Research, 18(1), 15-27. https://doi.org/10.1080/10503300701697513

Safran, J. D., \& Muran, J. C. (2006). Has the concept of the alliance outlived its usefulness? Psychotherapy, 43, 286-291. https://doi.org/10.1037/0033-3204.43.3.286

Schenk, N., Zimmermann, R., Fürer, L., Krause, M., Weise, S., Kaess, M., ... Schmeck, K. (2019). Trajectories of alliance ruptures in the psychotherapy of adolescents with borderline personality pathology: timing, typology and significance. Research in Psychotherapy: Psychopathology, Process and Outcome, 22(2), 199-211. https://doi.org/10.4081/ripppo.2019.348

Shirk, S., \& Karver, M. (2003). Prediction of treatment outcome from relationship variables in child and adolescent therapy: A metanalytic review. Journal of Consulting and Clinical Psychology, 71(3),452-464. https://doi.org/10.1037/0022$\underline{006 X .71 .3 .452}$

Stern, D. (1985). The interpersonal world of the infant: A view from psychoanalysis and developmental psychology. New York: Basic Books.

Weisz, J. R., Sandler, I. N., Durlak, J. A., \& Anton, B. S. (2005). Promoting and protecting youth mental health through evidence-based prevention and treatment. American Psychologist, 60(6), 628-648. https://doi.org/10.1037/0003-066X.60.6.628

Weisz, J. R., \& Kazdin, A. E. (Eds.). (2010). Evidence-based psychotherapies for children and adolescents. New York: Guilford Press. 